\title{
Plant growth and rhizome shoots of Agave angustifolia in different substrates, with fertigation and benzylaminopurine
}

\author{
Raquel Garnica-García ${ }^{1}$, José Raymundo Enríquez-del-Valle', Gerardo Rodríguez-Ortiz", \\ Isabel Pérez-León ${ }^{1}$, Ricardo Trejo-Calzada ${ }^{2}$, Isidro Morales ${ }^{3 *}$ \\ ${ }^{1}$ Tecnológico Nacional de México, División de Estudios de Posgrado e Investigación. Instituto Tecnológico del Valle de Oaxaca. Ex-Hacienda \\ de Nazareno, Xoxocotlán, Oaxaca. México. C.P. 71230. ²Unidad Regional de Zonas Áridas de la Universidad Autónoma Chapingo, Bermejillo, \\ Dgo. ${ }^{3}$ Instituto Politécnico Nacional, CIIDIR-Oaxaca, 71230, Oaxaca, México
}

\section{A B S TR A C T}

\begin{abstract}
The species Agave angustifolia can be propagated asexually with offshoots from rhizomes, bulbils from inflorescence, and through micropropagation to supply the growing demand for quality plant material for new plantations. In this investigation, Agave angustifolia plants of different initial sizes were evaluated for growth and emission of rhizome shoots, after 14 months under different conditions of substrate, fertigation and application of cytokinins. Bulbils were collected from inflorescences of plants at two sites and classified into two size categories: small $(\leq 7 \mathrm{~cm})$ and large $(>7 \mathrm{~cm})$. The bulbils were cultured for 14 months in pots with substrates that were mixtures of sand (A), soil (S), and bovine manure $(B m)(\%): 1) A+S(0.3: 0.7) ; 2) A+S+B m(0.3: 0.525: 0.175) ; 3) A+S+B m(0.3$ : $0.35: 0.35)$; 4) $A+S+B m(0.3: 0.175: 0.525)$. The plants in each substrate were separated into groups for application of different types of irrigation: 1) water; 2) $50 \%$ Steiner's nutritive solution, SNS-50\%; 3) SNS-50\% + $25 \mathrm{mg} \mathrm{L}^{-1}$ of benzylaminopurine. The results show that the emission of rhizome offshoots was affected by the substrate, type of irrigation and provenance of the plants. The plants in substrates 3 and 4 formed 2.2 rhizome offshoots, which was more than plants in the other substrates. The plants irrigated with water, SNS-50\%, and SNS-50\% + benzylaminopurine formed 1.1, 1.6 and 1.9 rhizome offshoots, respectively.
\end{abstract}

Keywords: Fertilization; Offshoot from rhizome; Substrate

\section{INTRODUCTION}

In the state of Oaxaca, Mexico, 25 species and varieties of cultivated and wild agaves are used for production of the distilled beverage called mescal (Espinosa et al., 2005). In the late 19th century and early 20th century, the process of selection and cultivation of mescal agaves began, outstanding of these agaves is $A$. angustifolia (Antonio and Terán, 2008). In 2014, the total cultivated area with agaves covered 8,587 ha where $A$. angustifolia occupied $77 \%$ to $95 \%$. This species is preferred because it is more precocious and has higher carbohydrate yield than other species (Nolasco, 2015). Depending on the region where this species is grown, its multiple uses make it agroecologically, economically and socially important (Berumen, 2009; García-Herrera et al., 2010; López et al., 2016). This plant is hardy, capable of growing and reproducing in shallow unfertile soils on steep slopes (Fig. 1A) with little water during several months of the year, whereas other crops do not thrive (Verduzco-Martínez et al., 2009). It also assimilates and transforms $\mathrm{CO}_{2}$ efficiently (García-Moya et al., 2011). In a study with Agave americana var oaxacensis, it was demonstrated that by improving nutritional supply through fertilizers, these plants achieved greater size (Enríquez-del Valle et al., 2013).

Agaves reproduce both sexually and asexually; thus they have a greater capacity to expand their distribution area (García-Mendoza, 2007). Agave angustifolia in the field reaches its sexual reproduction stage in seven to nine years (Antonio and Terán, 2008). Sexual reproduction is monocarpic because when seeds, fruits and bulbils are developing on the inflorescence, the gradual senescence of stem and leaves occurs, until the plant dies (Nobel, 1977; Szarek and Holmesley, 1996; Arrizaga and Ezcurra, 2002; Enríquez-del Valle, 2008). Asexual propagation occurs 


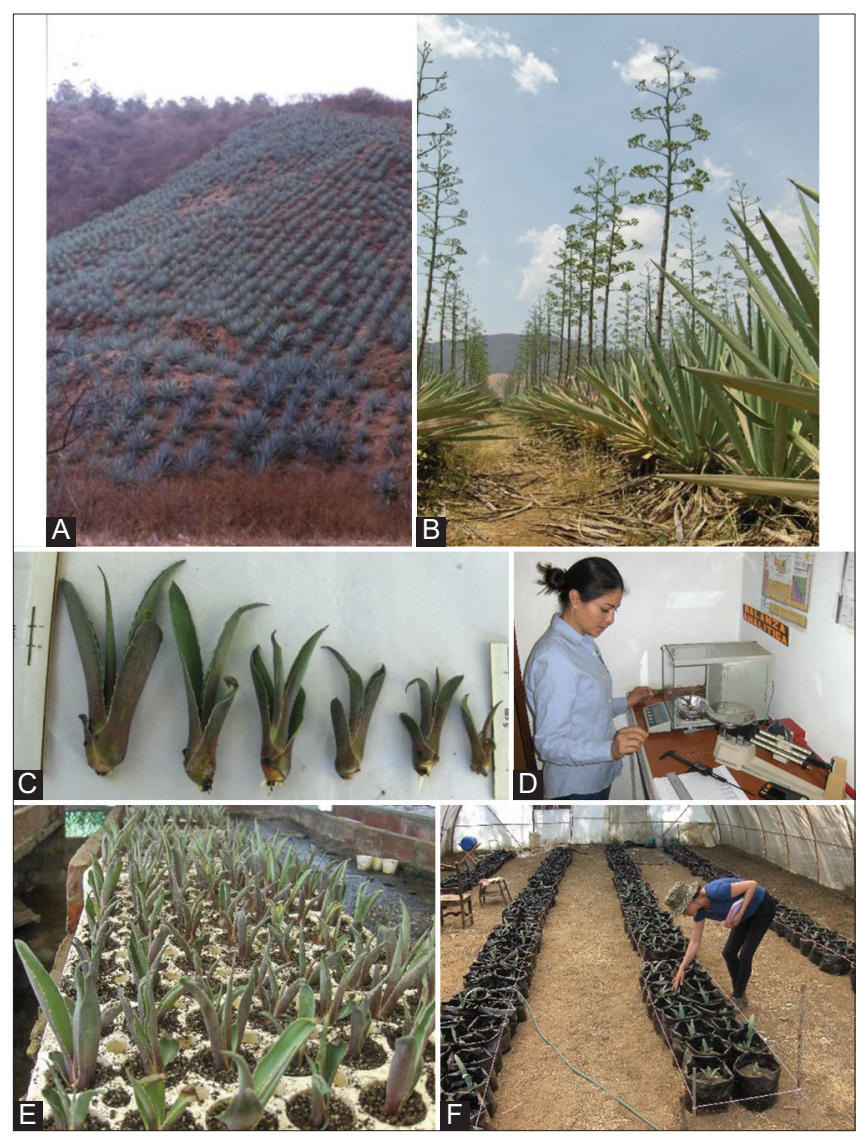

Fig 1. Images that describe the cultivation of the species and the experiment: A) Agave angustifolia plantation in steep slopes; B) Eight- to nine-year-old A. angustifolia plants used to produce bulbils, vegetative propagules; C) Bulbils of different sizes harvested from the inflorescence. The bulbils before establishing them in the substrate, these already have small roots at their base; D) The characteristics of the bulbili were described; E) The plants originated from bulbils were in trays with similar substrate, before establishing them to the treatments; F) Plants under different experimental conditions.

through rhizomes, which produce shoots and subsequently plants. The peasants use mainly this plant material for propagation because it is easy to obtain, and plantations can be established at low cost. The rhizome that originates from a vegetative bud at the lower part of the stem of mother plant, and grows horizontally underground, and when it emerges to the surface, it develops leaves and roots. When these new plants are more than $30 \mathrm{~cm}$ tall, they are separated from the mother plant to establish new plantations. A plant emits an average one to four rhizome shoots per year, and during its life cycle, it can produce up to 12 stems, depending on plant vigor and soil conditions. The young plants are generally planted definitively in the ground, but if they are less than $25 \mathrm{~cm}$ tall, they are established in a nursery for a period of six months or more until they are large enough to be planted definitively in the field (Espinosa et al., 2005; Enríquez-del Valle, 2008, García-Herrera et al., 2010). When the A. angustifolia plant in the field is seven to nine years old, it begins the development of the inflorescence (Fig. 1B) where it could develop numerous fruits with seeds. But when the flowers are close to the anthesis, they are injured at the level of the ovary, one by one, with a razor, which stimulates the development of vegetative propagules called bulbil. The vegetative propagules that are harvested from a plant are very heterogeneous in size (Fig. 1C), so they are classified in size categories to establish them in the nursery. Plants originating from bulbils of larger initial size remain in the nursery for six to 10 months, when they reach the appropriate size, greater than $30 \mathrm{~cm}$, to establish them in the field (Enríquez-del Valle, 2008). When an A. angustifolia plant is extracted from the nursery soil to take it to the field, it already shows the development of rhizomes that could be used in the propagation system.

The mature Agave angustifolia stem ("piña") contains a large amount of carbohydrates that are used as raw material for the production of mezcal and other distillates. When the plant is harvested, all the leaves are cut off, leaving the stem which is the centre of the rosette that looks like a pineapple, or piña. This is the part from which juice is extracted and processed into mescal. Demand for "piñas" is increasing (Zizumbo-Villareal et al., 2013), and new plantations of young plants $30 \mathrm{~cm}$ or more in size, of morphological quality, health and vigor are required (Martínez et al., 2013). Enríquez-del Valle et al. (2009), under nursery conditions, grew Agave angustifolia plants on 100\% compost substrate and fertigated with the Steiner's universal solution (Steiner, 1984) at 100\% nutrient concentration; they achieved larger plants that accumulated $37.8 \mathrm{~g}$ biomass and $2.2 \% \mathrm{~N}$ in the leaves. These quantities were 2.27 times the biomass and 1.27 times the $\mathrm{N}$ in leaves, compared with plants established in substrate with $25 \%$ compost $+75 \%$ agricultural soil and did not receive additional fertilization. It has been observed, but not documented, that the quantity of rhizome shoots emitted by plants with 1.5 years in nursery is directly related to vigor. To stimulate sprouting of more vegetative buds on the stem of mother plant and to develop more rhizomes, some exogenous cytokinin could be applied to the mother plant since it is known that this type of growth regulator stimulates sprouting of vegetative buds (Faiss et al., 1997; Mok et al., 1999). Cytokinins induces RNA processes and protein synthesis related to cell division and stem and leaf development (George et al., 2008). There is no information regarding the application of cytokinins to agave plants in nursery or in the field.

Increasing production of quality plants under nursery conditions would serve to develop specialized propagation programs and research aimed to increase productivity in Agave crops (Martínez-Aguilar and Peña-Álvarez, 2009; Palma et al., 2016). For this reason, this study evaluated growth and emission of rhizome shoots of Agave angustifolia 
plants from two provenances and of different sizes that were established in a nursery in substrates and supplied with fertigation and benzylaminopurine.

\section{MATERIALS AND METHODS}

\section{Obtaining bulbils (young plants)}

The study was conducted at the Technological Institute of the Valley of Oaxaca (ITVO) located in Santa Cruz Xoxocotlán, Oaxaca, México, at $17^{\circ} 02^{\prime} \mathrm{N}, 96^{\circ} 44^{\prime} \mathrm{W}$ and $1530 \mathrm{~m}$ altitude. The climate is arid and warm with summer rains. In October 2016, 384 bulbils were harvested from Agave angustifolia inflorescences at two different sites: 192 bulbils from one plant in the area of Candelaria Yegolé, Santa María Zoquitlan, Tlacolula, Oaxaca, located at $16^{\circ}$ 29' 37.7' $\mathrm{N}$ and $96^{\circ} 18^{\prime} 35.9^{\prime \prime} \mathrm{W}, 1211$ masl, with temperate and dry warm climate with annual precipitation between 300 to $500 \mathrm{~mm}$, mainly from late April to September, with average temperature of $22{ }^{\circ} \mathrm{C}$. Another 192 bulbils were harvested from one plant in the area of San Felipe del Agua, Oaxaca, located at $17^{\circ} 06^{\prime} 10.9^{\prime \prime} \mathrm{N}$ and $96^{\circ} 43^{\prime} 01.2$ of W, 1550 masl, with $22{ }^{\circ} \mathrm{C}$ average annual temperature and $727.6 \mathrm{~mm}$ annual precipitation (COPLADE, 2018).

\section{Experimental design, treatments and data analysis}

The bulbils were assessed on morphological characteristics (Fig. 1D), and then classified into two size categories: 1) 3 to $7 \mathrm{~cm}$, and 2) $>7$ to $>14 \mathrm{~cm}$. The bulbils were established in trays divided into 70 truncated inverted cone $182 \mathrm{~cm}^{3}$ cavities, with substrate prepared with peat moss and perlite in 1: 1 proportion (Fig. 1E). Under these conditions, the plants were kept for four months in a shade house with a metallic structure, translucent corrugated sheet roof, and translucent polyethylene side walls. Plants were irrigated daily from 11:00 to $15: 00 \mathrm{~h}$ by intermittent nebulization in periods of 10 seconds every 10 minutes.

The plants were transferred to a greenhouse with metallic structure and translucent white polyethylene cover. The plants were removed from the trays and individually established in $7 \mathrm{dm}^{3}$ black polyethylene bags (Fig. 1F), which contained different substrate mixtures of sand (A), soil $(\mathrm{S})$ and bovine manure $(\mathrm{Bm}): 1) \mathrm{A}+\mathrm{S}(0.3: 0.7) ; 2)$ $\mathrm{A}+\mathrm{S}+\mathrm{Bm}(0.3: 0.525: 0.175) ; 3) \mathrm{A}+\mathrm{S}+\mathrm{Bm}(0.3: 0.35$ : $0.35)$; 4) $\mathrm{A}+\mathrm{S}+\mathrm{Bm}(0.3$ : 0.175: 0.525). Physical and chemical characteristics of the substrates were determined by the procedures described in NOM-021-RECNAT-2000 (DOF, 2002).

Leaf and root samples were taken from 10 bulbils per plant from each provenance and were analyzed according to AOAC (2000), in the National Laboratory of Analysis of Plant, Water, Soil and Environment, CENID - RASPA of INIFAP, Gómez Palacio, Durango (Table 2).

All the plants from each provenance, by category of initial plant size, and type of substrate in which they were established, were separated into three groups to which different types of irrigation were applied: 1) water; 2) Steiner (1984) nutrient solution formulation, diluted to 50\% (SNS-50\%) and, 3) SNS-50\% $+25 \mathrm{mg} \mathrm{l}^{-1}$ benzylaminopurine. The plants received $500 \mathrm{~mL}$ of the corresponding irrigation type once a week for 14 months.

The experiment was established according to a completely randomized design with treatments in a $2 \times 2 \times 4 \times 3$ factorial arrangement (provenance of plants, initial size of plants, substrate in which they were established, type of irrigation), resulting in 48 treatments. The experimental unit was one plant and there were eight replications per treatment. After the experimental period, three plants were harvested from each treatment and separated into leaves, stem, root and rhizome offshoots. Number of leaves, roots and rhizome offshoots, length and width of the longest leaf $(\mathrm{cm})$, and plant height $(\mathrm{cm})$ were quantified. Volumes of leaf, stem and root $\left(\mathrm{cm}^{3}\right)$ of each plant were determined by immersion in a known volume of water in a graduated test tube. Subsequently, the leaves, stems and roots were placed separately in paper bags and dried in a convection oven at $90{ }^{\circ} \mathrm{C}$ for $120 \mathrm{~h}$, after which dry weight (g) of leaves, stem and root and total dry weight were quantified.

The data were analyzed to comply with homogeneity and normality of variances by means of the Bartlett and Shapiro-Wilk tests, respectively. The data that did not meet these assumptions were transformed with Log10 and subjected to an analysis of variance. For the comparison of means (Tukey, 0.05), untransformed data were used. Untransformed averages of number of shoots were compared using the Duncan test (0.05). In all the cases of statistical analysis routines, the software Statistical Analysis System was used (SAS Institute, 2004).

\section{RESULTS AND DISCUSSION}

The amount of essential nutrients was related to the amount of organic fertilizer incorporated into the substrates. The substrate that did not contain fertilizer had $2.2 \%$ organic matter, $\mathrm{MO}, 42.5 \mathrm{mg} \mathrm{N}-\mathrm{NO}_{3} \mathrm{~kg}^{-1}, 485.3 \mathrm{mg}$ $\mathrm{P} \mathrm{kg}^{-1}, 30.7 \mathrm{mg} \mathrm{Fe} \mathrm{kg}^{-1}, 3.3 \mathrm{mg} \mathrm{Zn} \mathrm{kg}^{-1}, 0.9 \mathrm{mg} \mathrm{B} \mathrm{kg}^{-1}, 5.2 \mathrm{mg}$ $\mathrm{Mg} \mathrm{kg}^{-1}, 145 \mathrm{mg}$ available $\mathrm{K} \mathrm{kg}^{-1}$. In contrast, the substrate whose volume was $52.5 \%$ organic fertilizer had $5.4 \% \mathrm{MO}$, 119.3 mg N-NO $\mathrm{kg}^{-1}, 2316.2 \mathrm{mg} \mathrm{P} \mathrm{kg}{ }^{-1}, 57.4 \mathrm{mg} \mathrm{Fe} \mathrm{kg}{ }^{-1}$, $8.9 \mathrm{mg} \mathrm{Zn} \mathrm{kg}{ }^{-1}, 1.7 \mathrm{mg} \mathrm{B} \mathrm{kg}^{-1}, 23.6 \mathrm{mg} \mathrm{Mg} \mathrm{kg}{ }^{-1}, 2637 \mathrm{mg}$ available $\mathrm{K} \mathrm{kg}^{-1}$. The electrical conductivity in substrate without fertilizer was $2.8 \mathrm{dS} \mathrm{m}^{-1}$, and when organic fertilizer 
was incorporated at rates of $17.5,35$ and $52.5 \%$ of the substrate volume, conductivity increased to 5.0, 7.4 and 10.8 $\mathrm{dS} \mathrm{m}^{-1}$, respectively, but $\mathrm{pH}$ did not vary from 8.4 of the substrate in which no fertilizer was incorporated (Table 1). For agave cultivation, there are still no defined standards for substrates (Crespo et al., 2013).

Plants grown from bulbils collected in Candelaria Yegolé and San Felipe del Agua were classified into two size categories, 3 to $7 \mathrm{~cm}$ and $>7 \mathrm{~cm}$ to $14 \mathrm{~cm}$. All the plants were placed in similar growth conditions for four months before starting the experiment. When the experiment started, they were $7.29,11.98,10.5$ and $16.08 \mathrm{~cm}$ in height, had 3.93, 6.13, 4.22 and 5.59 unfolded leaves, and had stems that were 1.71, $2.17,1.41$ and $2.05 \mathrm{~cm}$ in diameter, respectively. Nutrient contents in shoot and roots are shown in Table 2.

After 14 months of the experiment, 277 plants were harvested from the different treatments. They were of different sizes and developed different quantities of rhizomes. The analysis of variance (Table 3 ) showed that the factors substrate type (Fst) and initial plant size (Fips) had independent significant effects $(p \leq 0.05)$ on all the variables evaluated.
The factor irrigation type (Fit) had a significant effect ( $\mathrm{p}$ $\leq 0.05$ ) on most of variables, except stem (piña) diameter (SD), root dry weight (RDW) and root volume (RV). The factor provenance (Fpro) had significant effects ( $\mathrm{p} \leq 0.05$ ) on most of variables, except largest leaf length (LL), width (WL) and SD. The Fst $\times$ Fit interaction had a significant effect ( $\mathrm{p} \leq 0.05)$ on LL, plant height $(\mathrm{PH})$, total dry weight (TDW), leaf dry weight (LDW), RDW, stem dry weight (SDW), leaf volume (LV) and total volume (TV). The Fst $\times$ Fips interaction had a significant effect $(\mathrm{p} \leq 0.05)$ on number of roots (NR), LL, WL, PH, TDW, LDW, RDW, SDW, LV, SV, RV and TV. The Fst $\times$ Fpro interaction had a significant effect $(\mathrm{p} \leq 0.05)$ on NL, LL, TDW, LDW, SDW, LV, SV, RV and TV. The Fit $\times$ Fips interaction had a significant effect $(\mathrm{p} \leq 0.05)$ on TDW, SDW, SV and RV. The Fit $\times$ Fpro interaction had a significant effect $(\mathrm{p} \leq 0.05)$ on NL, LV and TV.

When the data were sorted by type of substrate (Table 4), it was observed that plant size had a positive relation to organic fertilizer content (up to $35 \%$ ) in the substrate. Plants in the substrate without fertilizer were $46.5 \mathrm{~cm}$ high and had 12.1 leaves; the largest leaf was $39.0 \mathrm{~cm}$ long and

Table 1: Physical and chemical characteristics of substrates.

\begin{tabular}{|c|c|c|c|c|c|c|c|c|c|}
\hline \multirow[t]{2}{*}{ Substrate } & PS & MO & $\mathrm{CO}_{3}$ & \multirow[t]{2}{*}{$\mathrm{pH}$} & \multirow{2}{*}{$\frac{C E}{d S ~ m^{-1}}$} & \multirow{2}{*}{$\frac{\mathrm{CIC}}{\mathrm{meq} / 100 \mathrm{~g}}$} & $\mathrm{~N}-\mathrm{NO}_{3}$ & $\mathbf{P}$ & KD \\
\hline & & $\%$ & & & & & \multicolumn{3}{|c|}{$\mathrm{mg} \mathrm{kg}^{-1}$} \\
\hline 1 & 36.6 & 2.2 & 0.6 & 8.4 & 2.8 & 6.6 & 42.5 & 485.3 & 145 \\
\hline 2 & 42.0 & 3.5 & 0.8 & 8.5 & 5.0 & 6.5 & 67.7 & 1029.2 & 765 \\
\hline 3 & 44.8 & 4.4 & 0.8 & 8.3 & 7.4 & 6.5 & 97.6 & 1939.0 & 1620 \\
\hline 4 & 43.3 & 5.4 & 0.8 & 8.4 & 10.8 & 7.4 & 119.3 & 2316.2 & 2637 \\
\hline \multirow[t]{2}{*}{ Substrate } & $\mathrm{Fe}$ & Mn & $\mathrm{Cu}$ & $\mathrm{Zn}$ & B & $\mathrm{Ca}$ & $\mathrm{Mg}$ & $\mathrm{Na}$ & $\mathbf{K}$ \\
\hline & \multicolumn{5}{|c|}{$\mathrm{mg} \mathrm{kg}^{-1}$} & \multicolumn{4}{|c|}{ Meq L ${ }^{-1}$} \\
\hline 1 & 30.7 & 58.5 & 4.8 & 3.3 & 0.9 & 19.2 & 5.2 & 3.8 & 0.6 \\
\hline 2 & 40.8 & 56.0 & 4.5 & 5.0 & 1.3 & 20.6 & 12.9 & 9.9 & 6.0 \\
\hline 3 & 50.6 & 65.3 & 4.8 & 6.3 & 1.5 & 20.1 & 18.8 & 17.1 & 17.6 \\
\hline 4 & 57.4 & 55.3 & 4.4 & 8.9 & 1.7 & 16.8 & 23.6 & 23.6 & 39.8 \\
\hline
\end{tabular}

S: substrate; PS: percentage of saturation; MO: organic matter; CO3: total carbonates; pH: hydrogen potential; CE: electrical conductivity; $\mathrm{N}-\mathrm{NO}_{3}$ : nitric nitrogen; KD: available potassium; P: available phosphorus; K: potassium available; Fe: iron; Mn: Manganese; Cu: copper; Zn: zinc; B: boron; Ca: calcium; Mg: magnesium; Na: sodium; K: potassium.

Table 2: Macronutrients and micronutrients in aerial part and root of bulbils-plants originated from inflorescence of Agave angustifolia.

\begin{tabular}{lcccc}
\hline & San Felipe (Aerial part) & Yegolé (Aerial part) & San Felipe (Root) & Yegolé (Root) \\
\hline Total nitrogen (\%) & 0.85 & 0.91 & 0.71 & 0.82 \\
Total phosphorus (\%) & 0.18 & 0.15 & 0.10 & 0.10 \\
Total calcium (\%) & 3.81 & 3.40 & 0.99 & 0.29 \\
Total magnesium (\%) & 0.82 & 0.68 & 0.24 & 0.27 \\
Total sodium (\%) & 0.19 & 0.16 & 0.63 & 0.17 \\
Total potassium (\%) & 1.24 & 0.88 & 630.00 & 0.75 \\
Total iron $\left(\mathrm{mg} \mathrm{kg}^{-1}\right)$ & 368.00 & 264.60 & 18.90 & 1.90 \\
Total manganese $\left(\mathrm{mg} \mathrm{kg}^{-1}\right)$ & 15.50 & 10.70 & 180.50 & 18.10 \\
Copper $\left(\mathrm{mg} \mathrm{kg}^{-1}\right)$ & 16.00 & 16.10 & 11.80 & 47.80 \\
Zinc $\left(\mathrm{mg} \mathrm{kg}^{-1}\right)$ & 38.90 & 40.20 & 0.01 & 60.10 \\
Boron $\left(\mathrm{mg} \mathrm{kg}^{-1}\right)$ & 0.01 & 0.01 & 0.01 \\
\hline
\end{tabular}


Table 3: Summary of analysis of variance in morphological characteristics of Agave angustifolia plants in substrates and different nutritional supply.

\begin{tabular}{|c|c|c|c|c|c|c|c|c|c|}
\hline \multirow[t]{2}{*}{ SV } & \multirow[t]{2}{*}{ DF } & \multicolumn{8}{|c|}{ Square means and significance } \\
\hline & & NL & NR & LL & WL & DP & HP & & TDW \\
\hline Fst & 3 & $0.022^{\star *}$ & $21.75^{\star}$ & $0.026^{* *}$ & $2.676^{* *}$ & $0.049^{\star *}$ & $316.64^{\star *}$ & & $8329.85^{\star *}$ \\
\hline Fit & 2 & $0.036^{* *}$ & $45.05^{\star *}$ & $0.027^{* *}$ & $1.252^{\star *}$ & $0.008^{\text {ns }}$ & $569.03^{\star *}$ & & $3901.65^{\star *}$ \\
\hline Fips & 1 & $0.084^{* *}$ & $117.36^{* *}$ & $0.080^{\star *}$ & $1.969^{\star *}$ & $0.045^{*}$ & $1019.77^{* *}$ & & $15748.37^{\star *}$ \\
\hline Fpro & 1 & $0.088^{* *}$ & $81.00^{* *}$ & $0.003^{\text {ns }}$ & $0.027^{\text {ns }}$ & $0.000^{\text {ns }}$ & $45.11^{*}$ & & $13605.86^{* *}$ \\
\hline Fst $\times$ Fit & 6 & $0.004^{\text {ns }}$ & $9.63^{\text {ns }}$ & $0.005^{* *}$ & $0.367^{\text {ns }}$ & $0.010^{\text {ns }}$ & $49.00^{* *}$ & & $886.71^{*}$ \\
\hline Fst $\times$ Fips & 3 & $0.004^{\mathrm{ns}}$ & $38.12^{\star *}$ & $0.004^{* *}$ & $0.877^{\star *}$ & $0.013^{\text {ns }}$ & $40.29^{* *}$ & & $2537.62^{\star *}$ \\
\hline FstxFpro & 3 & $0.011^{\star \star}$ & $1.06^{\mathrm{ns}}$ & $0.002^{*}$ & $0.058^{\text {ns }}$ & $0.013^{\text {ns }}$ & $12.18^{\text {ns }}$ & & $887.82^{\star}$ \\
\hline FitxFips & 2 & $0.004^{\text {ns }}$ & $7.92^{\text {ns }}$ & $0.001^{\text {ns }}$ & $0.059^{\text {ns }}$ & $0.015^{\mathrm{ns}}$ & $9.06^{\text {ns }}$ & & $1551.88^{* *}$ \\
\hline Fit×Fpro & 2 & $0.007^{*}$ & $4.69^{\text {ns }}$ & $0.001^{\text {ns }}$ & $0.136^{\text {ns }}$ & $0.008^{\mathrm{ns}}$ & $17.74^{\text {ns }}$ & & $907.18^{\text {ns }}$ \\
\hline Fips $\times$ Fpro & 1 & $0.000^{\text {ns }}$ & $7.11^{\mathrm{ns}}$ & $0.000^{\text {ns }}$ & $0.069^{\text {ns }}$ & $0.008^{\text {ns }}$ & $10.67^{\text {ns }}$ & & $102.84^{\text {ns }}$ \\
\hline FstxFitxFips & 6 & $0.003^{\text {ns }}$ & $17.99^{\star *}$ & $0.001^{\text {ns }}$ & $0.105^{\mathrm{ns}}$ & $0.008^{\text {ns }}$ & $9.03^{\text {ns }}$ & & $988.84^{\star *}$ \\
\hline Fst $\times$ Fit $\times$ Fpro & 6 & $0.000^{\text {ns }}$ & $4.49^{\text {ns }}$ & $0.002^{*}$ & $0.204^{\mathrm{ns}}$ & $0.008^{\mathrm{ns}}$ & $13.10^{\text {ns }}$ & & $986.84^{* *}$ \\
\hline Fit×Fips×Fpro & 2 & $0.002^{\text {ns }}$ & $1.17^{\mathrm{ns}}$ & $0.000^{\text {ns }}$ & $0.005^{\mathrm{ns}}$ & $0.007^{\text {ns }}$ & $2.37^{\mathrm{ns}}$ & & $113.08^{\text {ns }}$ \\
\hline Fst $\times$ Fips $\times$ Fpro & 3 & $0.001^{\text {ns }}$ & $16.57^{*}$ & $0.002^{\text {ns }}$ & $0.217^{\mathrm{ns}}$ & $0.011^{\mathrm{ns}}$ & $8.44^{\mathrm{ns}}$ & & $441.09^{n s}$ \\
\hline Fst $\times$ FitxFips $\times$ Fpro & 6 & $0.001^{\text {ns }}$ & $2.61^{\mathrm{ns}}$ & $0.000^{\text {ns }}$ & $0.267^{\text {ns }}$ & $0.014^{\mathrm{ns}}$ & $5.85^{\text {ns }}$ & & $315.46^{\text {ns }}$ \\
\hline Error & 96 & 0.002 & 5.74 & 0.000 & 0.175 & 0.009 & 6.63 & & 312.35 \\
\hline Total & 143 & & & & & & & & \\
\hline \multirow[t]{2}{*}{ sv } & GL & \multicolumn{8}{|c|}{ Square means and significance } \\
\hline & & FDW & RDW & PDW & LV & PV & RV & TV & NO \\
\hline Fst & 3 & $0.117^{\star *}$ & $0.412^{* \star}$ & $844.89^{* *}$ & $0.602^{* *}$ & $0.243^{\star *}$ & $0.552^{* *}$ & $0.474^{* *}$ & $1.133^{\star *}$ \\
\hline Fit & 2 & $0.165^{\star *}$ & $0.011^{\mathrm{ns}}$ & $210.66^{*}$ & $0.279^{\star *}$ & $0.168^{\star \star}$ & $0.000^{\text {ns }}$ & $0.199^{* *}$ & $0.284^{* *}$ \\
\hline Fips & 1 & $0.404^{\star \star}$ & $0.145^{\star \star}$ & $1738.13^{\star *}$ & $0.517^{\star *}$ & $0.544^{\star \star}$ & $0.126^{\star *}$ & $0.484^{* *}$ & $0.104^{\text {ns }}$ \\
\hline Fpro & 1 & $0.184^{\star *}$ & $0.133^{\star *}$ & $2658.52^{* *}$ & $0.060^{* *}$ & $0.178^{\star *}$ & $0.080^{*}$ & $0.092^{* *}$ & $2.348^{* *}$ \\
\hline Fst $\times$ Fit & 6 & $0.017^{*}$ & $0.018^{*}$ & $141.06^{*}$ & $0.026^{* *}$ & $0.006^{\mathrm{ns}}$ & $0.028^{\text {ns }}$ & $0.019^{* *}$ & $0.045^{\text {ns }}$ \\
\hline Fst $\times$ Fips & 3 & $0.035^{\star *}$ & $0.046^{\star *}$ & $392.70^{\star *}$ & $0.040^{* *}$ & $0.039^{*}$ & $0.066^{\star *}$ & $0.042^{* *}$ & $0.103^{*}$ \\
\hline FstxFpro & 3 & $0.022^{*}$ & $0.018^{\mathrm{ns}}$ & $439.94^{\star *}$ & $0.014^{*}$ & $0.056^{\star \star}$ & $0.134^{\star *}$ & $0.012^{*}$ & $0.209^{* *}$ \\
\hline Fit×Fips & 2 & $0.009^{\text {ns }}$ & $0.045^{\mathrm{ns}}$ & $643.95^{\star *}$ & $0.002^{\mathrm{ns}}$ & $0.047^{*}$ & $0.071^{*}$ & $0.011^{\text {ns }}$ & $0.008^{\text {ns }}$ \\
\hline FitxFpro & 2 & $0.002^{\text {ns }}$ & $0.017^{\mathrm{ns}}$ & $116.70^{\text {ns }}$ & $0.022^{*}$ & $0.023^{\text {ns }}$ & $0.019^{\text {ns }}$ & $0.023^{* *}$ & $0.083^{\text {ns }}$ \\
\hline Fips $\times$ Fpro & 1 & $0.003^{\text {ns }}$ & $0.000^{\text {ns }}$ & $9.65^{\text {ns }}$ & $0.002^{\mathrm{ns}}$ & $0.001^{\mathrm{ns}}$ & $0.015^{\text {ns }}$ & $0.003^{\text {ns }}$ & $0.154^{*}$ \\
\hline FstxFitxFips & 6 & $0.028^{\star *}$ & $0.011^{\mathrm{ns}}$ & $130.68^{*}$ & $0.015^{\star *}$ & $0.013^{\text {ns }}$ & $0.022^{\text {ns }}$ & $0.012^{* *}$ & $0.066^{\text {ns }}$ \\
\hline Fst $\times$ Fit $\times$ Fpro & 6 & $0.038^{\star *}$ & $0.012^{\mathrm{ns}}$ & $97.77^{\text {ns }}$ & $0.008^{\text {ns }}$ & $0.011^{\mathrm{ns}}$ & $0.017^{\mathrm{ns}}$ & $0.008^{\text {ns }}$ & $0.011^{\mathrm{ns}}$ \\
\hline Fit×Fips xFpro & 2 & $0.003^{\text {ns }}$ & $0.000^{\text {ns }}$ & $23.81^{\text {ns }}$ & $0.002^{\text {ns }}$ & $0.012^{\text {ns }}$ & $0.010^{\text {ns }}$ & $0.001^{\text {ns }}$ & $0.030^{\text {ns }}$ \\
\hline Fst $\times$ Fips $\times$ Fpro & 3 & $0.016^{\mathrm{ns}}$ & $0.003^{\text {ns }}$ & $98.57^{\text {ns }}$ & $0.008^{\text {ns }}$ & $0.003^{\text {ns }}$ & $0.001^{\mathrm{ns}}$ & $0.004^{\text {ns }}$ & $0.182^{* *}$ \\
\hline Fst $\times$ FitxFips $\times$ Fpro & 6 & $0.019^{*}$ & $0.009^{\text {ns }}$ & $21.71^{\mathrm{ns}}$ & $0.008^{\text {ns }}$ & $0.003^{\text {ns }}$ & $0.017^{\mathrm{ns}}$ & $0.006^{n s}$ & $0.027^{\text {ns }}$ \\
\hline Error & 96 & 0.006 & 0.007 & 56.11 & 0.004 & 0.010 & 0.015 & 0.004 & 0.037 \\
\hline Total & 143 & & & & & & & & \\
\hline
\end{tabular}

SV: sources of variation; DF: degrees of freedom; Fst: Substrate; Fit: Irrigation; Fips: Initial plant size; Fpro: Provenance; NL: Number of leaves; LL: length of leaf; WL: width of leaf; FDW: Foliar dry weight; RDW: Root dry weight; TV: Total volume; PV: Pineapple volume; RV: Root volume; LV: Leaf volume; HP: Height of plant; NR: Number of roots; TDW: Total dry weight; PDW: pineapple dry weight; DP: diameter of pineapple; NO: Number of offsprings; ns: non-significant values ( $p>0.05)$; ${ }^{*}$ values with significant effects $(p \leq 0.05)$, ${ }^{* *}$ values with highly significant effects $(p \leq 0.01)$.

$3.7 \mathrm{~cm}$ wide. Stem dry weight was $29.2 \mathrm{~g}$, root dry weight was $14.7 \mathrm{~g}$, leaf dry weight was $49.8 \mathrm{~g}$, and total dry weight was $84.8 \mathrm{~g}$. They had 21.0 roots, $116.2 \mathrm{~cm}^{3}$ stem volume, $40.63 \mathrm{~cm}^{3}$ root volume, $312.5 \mathrm{~cm}^{3}$ leaf volume, and $5.7 \mathrm{~cm}$ stem diameter. In contrast, plants in substrate with $35 \%$ organic fertilizer had a height of $53.6 \mathrm{~cm}$ and 13.8 leaves; largest leaf was $44.5 \mathrm{~cm}$ long and $4.4 \mathrm{~cm}$ wide, stem dry weight was $35.7 \mathrm{~g}, 26.1 \mathrm{~g}$ root dry weight, $54.2 \mathrm{~g}$ leaf dry weight, and $116.1 \mathrm{~g}$ total dry weight. They had 22.8 roots, $179.1 \mathrm{~cm}^{3}$ stem volume, $87.2 \mathrm{~cm}^{3}$ root volume, $615.0 \mathrm{~cm}^{3}$ leaf volume, and $6.5 \mathrm{~cm}$ stem diameter. All the values of these variables were significantly different between the two treatments (Tukey, 0.05). The variables of plants established in the substrate with more than 35\% organic fertilizer did not differ significantly. When the data were sorted by type of irrigation supplied to the plants, it was observed that the fertigated plants were significantly larger (Tukey, 0.05) in PH, NL, LL, WL, SDW, TDW, LDW, TV, NR, SV, SD and LV than plants irrigated with only water. When the data were ordered by plant origin, it is observed that those plants collected in San Felipe del Agua were significantly (Tukey, 0.05) larger in PH, NL, SDW, RDW, TDW, LDW, TV, NR, SV, RV and LV than the plants from Yegolé.

When the data were sorted by plant initial size, at the end of the experiment there were no significant differences 
Garnica-García, et al.

Table 4: Characteristics of Agave angustifolia plants from two provenances and two initial size categories, which during 14 months in nursery were subjected to different substrata and nutritional supply.

\begin{tabular}{|c|c|c|c|c|c|c|c|c|}
\hline Fac & $\mathbf{N}$ & LL (cm) & WL (cm) & PDW (g) & RDW (g) & TDW (g) & FDW (g) & TV $\left(\mathrm{cm}^{3}\right)$ \\
\hline \multirow[t]{4}{*}{ Fst } & 1 & $39.0 \pm 4.3 b$ & $3.7 \pm 0.5 b$ & $29.2 \pm 12.8 b$ & $14.7 \pm 3.5 d$ & $84.8 \pm 26.7 b$ & $40.8 \pm 13.5 b$ & $469.4 \pm 129.5 c$ \\
\hline & 2 & $42.9 \pm 5.2 \mathrm{a}$ & $4.0 \pm 0.4 b$ & $36.4 \pm 11.6 \mathrm{a}$ & $23.4 \pm 6.3 b$ & $112.5 \pm 32.3 a$ & $52.6 \pm 18.4 a$ & $708.2 \pm 194.0 b$ \\
\hline & 3 & $44.5 \pm 4.2 \mathrm{a}$ & $4.4 \pm 0.4 a$ & $35.7 \pm 10.8 \mathrm{a}$ & $26.1 \pm 6.2 a$ & $116.1 \pm 30.0 a$ & $54.2 \pm 15.5 a$ & $881.5 \pm 265.9 a$ \\
\hline & 4 & $44.4 \pm 5.1 a$ & $4.3 \pm 0.4 a$ & $26.6 \pm 7.9 b$ & $20.4 \pm 3.6 c$ & $92.3 \pm 16.5 b$ & $45.3 \pm 7.9 \mathrm{~b}$ & $727.6 \pm 139.3 b$ \\
\hline \multirow[t]{3}{*}{ Fit } & W & $40.2 \pm 6.3 b$ & $3.9 \pm 0.4 b$ & $29.8 \pm 11.3 b$ & $20.4 \pm 6.8 a$ & $91.9 \pm 27.8 b$ & $41.6 \pm 13.3 \mathrm{c}$ & $595.0 \pm 235.5 b$ \\
\hline & SNS & $43.7 \pm 3.7 a$ & $4.2 \pm 0.6 a$ & $34.0 \pm 10.8 a$ & $21.8 \pm 6.6^{\mathrm{a}}$ & $109.7 \pm 30.8 \mathrm{a}$ & $53.9 \pm 17.1 \mathrm{a}$ & $753.1 \pm 231.8 a$ \\
\hline & SNS+BA & $44.2 \pm 4.3 a$ & $4.2 \pm 0.4 a$ & $32.2 \pm 12.6 a$ & $21.3 \pm 6.4^{\mathrm{a}}$ & $102.7 \pm 28.7 a$ & $49.1 \pm 12.4 b$ & $742.9 \pm 220.9 a$ \\
\hline \multirow[t]{2}{*}{ Fpro } & $\mathrm{Y}$ & $42.2 \pm 4.8 a$ & $4.1 \pm 0.5 a$ & $27.7 \pm 10.9 b$ & $19.7 \pm 5.9 b$ & $91.7 \pm 26.9 b$ & $44.2 \pm 13.2 b$ & $651.6 \pm 209.6 b$ \\
\hline & SF & $43.2 \pm 5.5 a$ & $4.1 \pm 0.5 a$ & $36.3 \pm 10.7 a$ & $22.6 \pm 6.9 a$ & $111.2 \pm 29.7 a$ & $52.2 \pm 16.0 \mathrm{a}$ & $741.7 \pm 259.3 a$ \\
\hline \multirow[t]{2}{*}{ Fips } & $\mathrm{Sm}$ & $40.5 \pm 5.3 b$ & $4.0 \pm 0.4 b$ & $28.5 \pm 10.3 b$ & $19.7 \pm 6.0 b$ & $91.0 \pm 26.8 b$ & $42.7 \pm 13.4 b$ & $605.5 \pm 195.4 b$ \\
\hline & $\mathrm{B}$ & $44.9 \pm 4.0 \mathrm{a}$ & $4.2 \pm 0.5 a$ & $35.5 \pm 11.9 \mathrm{a}$ & $22.6 \pm 6.8 \mathrm{a}$ & $111.9 \pm 29.3 a$ & $53.7 \pm 14.9 a$ & $787.9 \pm 245.4 a$ \\
\hline Fac & $\mathbf{N}$ & $\mathrm{PH}(\mathrm{cm})$ & NR & PV $\left(\mathrm{cm}^{3}\right)$ & NL & $\mathrm{RV}\left(\mathrm{cm}^{3}\right)$ & $\mathrm{DP}(\mathrm{cm})$ & $\operatorname{LV}\left(\mathrm{cm}^{3}\right)$ \\
\hline \multirow[t]{4}{*}{ Fst } & 1 & $46.6 \pm 5.5 c$ & $21.0 \pm 2.3 b$ & $116.2 \pm 41.2 b$ & $12.1 \pm 2.0 \mathrm{~b}$ & $40.63 \pm 10.1 c$ & $5.7 \pm 0.8 c$ & $312.5 \pm 95.0 \mathrm{~d}$ \\
\hline & 2 & $51.5 \pm 5.7 b$ & $21.3 \pm 3.4 b$ & $160.8 \pm 48.9 a$ & $13.4 \pm 1.9 a$ & $71.8 \pm 32.3 a b$ & $6.2 \pm 0.7 a b$ & $475.6 \pm 139.6 \mathrm{c}$ \\
\hline & 3 & $53.6 \pm 5.1 a$ & $22.8 \pm 3.0 a$ & $179.1 \pm 58.9 a$ & $13.8 \pm 2.5^{\mathrm{a}}$ & $87.2 \pm 75.5 a$ & $6.5 \pm 0.6 a$ & $615.0 \pm 181.2 a$ \\
\hline & 4 & $50.9 \pm 3.3 b$ & $21.5 \pm 2.9 b$ & $135.2 \pm 30.4 b$ & $13.5 \pm 1.3 a$ & $57.0 \pm 12.4 \mathrm{bc}$ & $5.9 \pm 0.5 b c$ & $535.2 \pm 108.4 b$ \\
\hline \multirow[t]{3}{*}{ Fit } & W & $46.7 \pm 5.4 b$ & $20.5 \pm 3.3 b$ & $126.1 \pm 44.1 b$ & $12.2 \pm 1.7 b$ & $69.2 \pm 70.2 \mathrm{a}$ & $5.71 \pm 0.7 b$ & $398.6 \pm 154.6 b$ \\
\hline & SNS & $52.5 \pm 4.6 a$ & $22.2 \pm 2.9 a$ & $160.2 \pm 55.6 a$ & $13.8 \pm 2.1^{a}$ & $61.2 \pm 21.9 a$ & $6.3 \pm 0.6 a$ & $531.5 \pm 169.0 a$ \\
\hline & SNS+BA & $52.8 \pm 4.5 a$ & $22.2 \pm 2.4 a$ & $157.2 \pm 48.1 \mathrm{a}$ & $13.5 \pm 2.1^{\mathrm{a}}$ & $62.0 \pm 26.0 \mathrm{a}$ & $6.3 \pm 0.6 a$ & $523.6 \pm 168.4 a$ \\
\hline \multirow[t]{2}{*}{ Fpro } & $\mathrm{Y}$ & $50.1 \pm 5.8 b$ & $20.9 \pm 2.9 b$ & $135.9 \pm 46.3 b$ & $12.5 \pm 1.9 b$ & $57.1 \pm 20.0 b$ & $6.0 \pm 0.7 a$ & $458.6 \pm 160.9 b$ \\
\hline & SF & $51.2 \pm 5.3 a$ & $22.4 \pm 2.8 \mathrm{a}$ & $159.8 \pm 54.0 \mathrm{a}$ & $13.9 \pm 1.9 a$ & $71.2 \pm 59.6 \mathrm{a}$ & $6.2 \pm 0.7 \mathrm{a}$ & $510.6 \pm 183.7 a$ \\
\hline \multirow[t]{2}{*}{ Fips } & $\mathrm{Sm}$ & $48.0 \pm 5.0 b$ & $20.7 \pm 2.7 b$ & $127.6 \pm 42.1 b$ & $12.4 \pm 1.5 b$ & $57.0 \pm 18.9 b$ & $5.9 \pm 0.6 b$ & $420.7 \pm 148.2 b$ \\
\hline & B & $53.3 \pm 4.8 a$ & $22.5 \pm 3.0 \mathrm{a}$ & $168.1 \pm 52.4 a$ & $14.4 \pm 2.2 \mathrm{a}$ & $71.2 \pm 59.9 a$ & $6.3 \pm 0.8 a$ & $548.4 \pm 175.5 a$ \\
\hline
\end{tabular}

Fac: factor; N: level; Fst: substrate; Fit: irrigation; W: water; SNS: Steiner's nutritive solution; BA: bencilaminopurine; Fips: initial plant size; Sm: Small; B: Big); Fpro: provenance; Y: Yegolé; SF: San Felipe; NL: number of leaves; LL: leaf length; LW: leaf width; PDW: pineapple dry weight; RDW: root dry weight; TDW: total dry weight; FDW: leaf dry weight; TV: total volume; PH: plant height; NR: number of roots; PV: pineapple volume; NL: number of leaves; RV: root volume; PD: pineapple diameter; LV: leaf volume. Values with the same letter in the same row are not significantly different (Tukey; 0.05). The mean is accompanied by the standard deviation.

(Tukey, 0.05) between those initially classified as small and those classified as large in all the evaluated characteristics (Table 4). The plants fertigated with SNS 50\% had a larger increase $(\mathrm{p}<0.0001)$ in the variables LL, WL, SDW, TDW, FDW, TV, PH, NR, SV, NL, SD and LV, than the plants that were not fertigated (Table 4). These results coincide with those obtained by Martínez-Ramírez et al. (2013), who found that $A$. angustifolia and $A$. potatorum plants fertilized with $30 \mathrm{~N}-20 \mathrm{P}-15 \mathrm{~K} \mathrm{ha}^{-1}$ under field conditions showed more growth and accumulation of biomass than non-fertilized plants. Zuñiga et al. (2018) evaluated Agave tequilana plants fertigated during a complete 77 -month crop cycle and found that the fertilized plants accumulated more total dry matter and up to five times the dry matter in the stem and 5.3 times the total reducing sugars compared to unfertilized plants. According to Nobel (1989), adequate nutritional supply improves agave physiological condition which increases $\mathrm{CO}_{2}$ fixation efficiency and biomass productivity.

The plants that were established in substrate $3(30 \%$ sand + $35 \%$ soil $+35 \%$ organic fertilizer) and in substrate $4(30 \%$ sand $+17.5 \%$ soil $+52.5 \%$ organic fertilizer), developed 2.20 rhizome offshoots, a significantly (Duncan, 0.05) larger number than the 0.6 offshoots developed by plants in the substrate in which no organic fertilizer was incorporated (Fig. 2). This response could be attributed to the fact that the plants had more nutrients available in substrates 3 and 4 , providing appropriate conditions for plant growth and emission of rhizome shoots.

Substrates 3 and 4 had 4.4 and 5.4\% organic matter, respectively. These percentages are considered high in the classification of non-volcanic soils according to NOM021-RECNAT-2000 (DOF, 2002). Studies conducted by Crespo et al. (2013) found that agave plants that were established in substrates with $50 \%$ compost developed piñas with a $40 \%$ larger diameter those from plants that were in substrate without compost. Each rhizome offshoot originates from the sprouting of a vegetative bud on the stem, and the amount of nutrients in the stem and leaves could have a positive relationship with the amount of buds induced to sprout. The plants irrigated with SNS-50\%, or with SNS-50\% + benzylaminopurine developed 1.6 and 1.9 rhizome offshoots, significantly higher (Duncan, 0.05) than the 1.1 shoots emitted by plants irrigated with only water. The substrate does not provide the plant with sufficient essential nutrients for optimum growth, but it can 


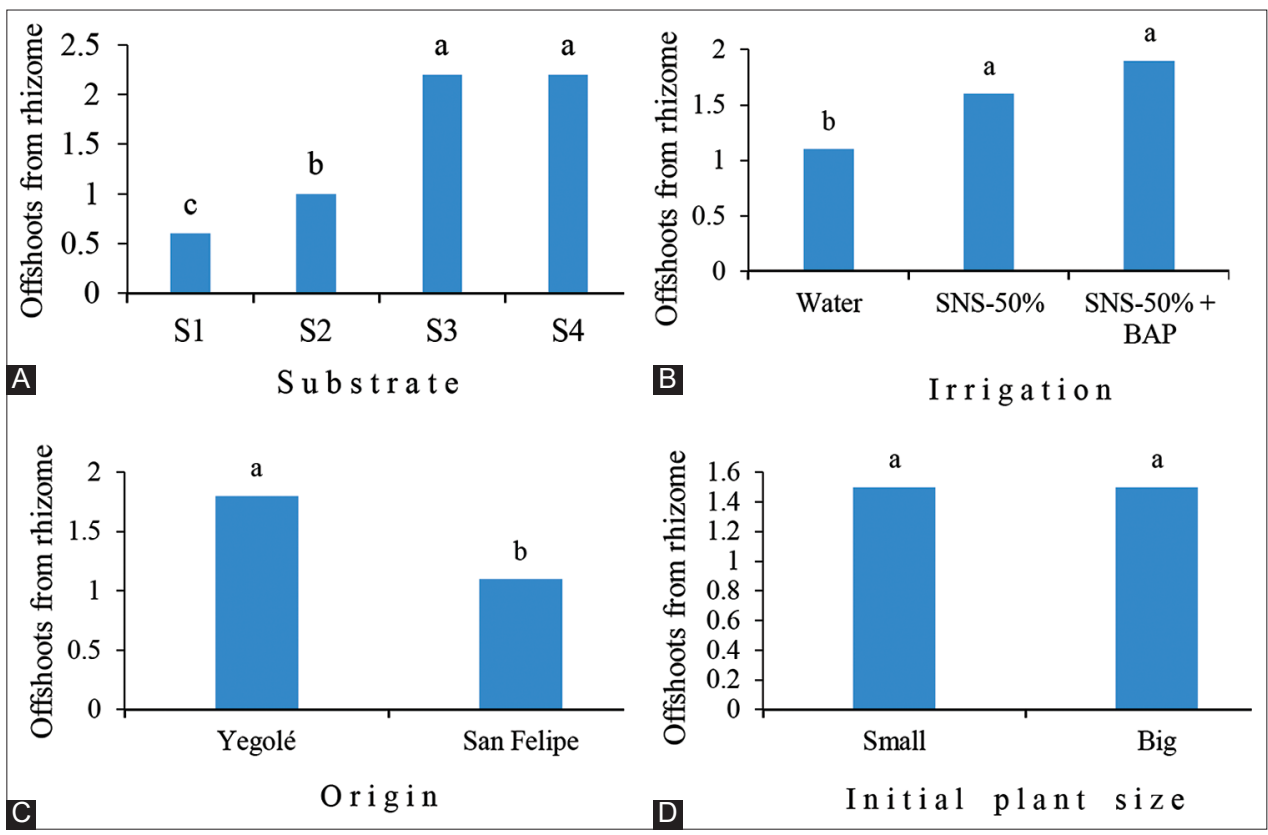

Fig. 2: Development of offshoots from rhizome in plants of Agave angustifolia with different: A) substrates, B) irrigation type, C) origin and D) initial size during 14 months in the nursery. In each factor, bars with different letters indicate significant differences (Duncan, 0.05).

be supplemented with mineral fertilization that provides nutrients readily available to the plant. The above coincides with Trejo-Téllez et al. (2013) in that alcatraz (Zantedeschia aethiopica), plants increased emission of suckers in response to the level of nutrient supply during the vegetative stage. In relation to the present work, there are no references of ex vitro application cytokinins to Agave plants to stimulate sprouting of vegetative buds. For the in vitro propagation of Agave americana (Miguel et al., 2014) and Agave angustifolia (Ríos-Ramírez et al., 2017) the data show that the cytokinins stimulated the development of multiple shoots.

The bulbil plants collected from mother plants in the community of Candelaria Yegolé generated more rhizome offshoots $(p<0.05)$ than those from the community of San Felipe del Agua. However, the Yegolé plants reached a smaller average size than the plants from San Felipe. This suggests topics for future studies: 1) differences in bulbil plant vigor; 2) genetic variability of plants from which the bulbils were harvested that reached different sizes after 14 months (Trame et al., 1995; Silva-Montellano and Eguiarte, 2003), the possible genetic differences of plants of the same plant species with different provenances that could be preserved by vegetative propagation (Infante et al., 2003; Barraza-Morales et al., 2006).

For vegetative propagation of agaves, data obtained by Lara-Ávila and Alpuche-Solís (2016) reveals that in A. salmiana ssp crassispina $88.73 \%$ of total genetic variation is between individuals, while $11.27 \%$ is between populations. Also, $A$. angustifolia and $A$. victoriae-reginae possess high degrees of intra-species genetic diversity and differentiation between populations (Eguiarte et al., 2013). On the other hand, the initial size of the plants did not influence the quantity of rhizome offshoots emitted (Fig. 2).

\section{CONCLUSION}

Agave angustifolia plants that were in the substrate that had from 17.5 to $35 \%$ of organic fertilizer, in relation to its volume, had $4.4 \%$ and $5.4 \%$ of organic matter, and higher contents of the nutrients $\mathrm{N}, \mathrm{P}, \mathrm{K}, \mathrm{Fe}, \mathrm{Zn}, \mathrm{B}$ and $\mathrm{Mg}$ than the substrate in which no organic fertilizer was incorporated. In the substrates with organic fertilizer, Agave angustifolia plants grew taller and had larger number of leaves, leaf size, leaf volume, root volume, stem volume, leaf volume, root volume and total dry weight. They also developed more rhizome offshoots than plants that were in substrate in which no organic fertilizer was incorporated. Fertigated Agave angustifolia plants and those that received fertigation plus cytokinins emitted a larger number of offshoots than plants that received only water.

\section{ACKNOWLEDGMENTS}

The authors thank the National Technological Institute of Mexico and the National Council of Science and Technology for the support received for the present investigation. To the Regional Unit of Arid Zones of the Autonomous University Chapingo and to the National Laboratory of Analysis of Plant, Water, Soil and Environment CENID-RASPA of INIFAP, Gómez Palacio, Durango, for their support in the analysis of plant material and substrates. 


\section{Author's contributions}

Raquel Garnica-García conducted the experiments and wrote the first manuscript, José Raymundo Enríquez-del Valle designed the experiments and participated in the laboratory work and the data analysis, Gerardo RodríguezOrtiz, Isabel Pérez-León and Ricardo Trejo-Calzada and Isidro Morales, review the manuscript, and were responsible for the final review of the manuscript. All authors read and approved the final manuscript.

\section{REFERENCES}

AOAC. 2000. Official Methods of Analysis. $17^{\text {th }}$ ed. Official Methods of Analysis of AOAC International. CAB Publisher, Gaithersburg, MD.

Antonio, B. J. and M. E. Terán. 2008. Estrategias de producción y mercadotecnia del mezcal en Oaxaca. El Cotidiano. 23 1-7.

Arrizaga, S. and E. Ezcurra. 2002. Propagation mechanisms in Agave macroacantha (agavaceae), a tropical arid-land succulent rosette. Am. J. Bot. 89 632-641.

Barraza-Morales, A., F. L. Sánchez-Teyer, M. Robert. M. Esqueda and A. Gardea. 2006. Variabilidad genética en Agave angustifolia Haw de la sierra sonorense, México, determinada con marcadores AFLP. Revista Fitotecnia. 29 1-8.

Berumen, B. M. E. 2009. Oaxaca la Actividad Productiva Magueymezcal. Oaxaca, México, p. 87.

COPLADE. 2018. Coordinación General del Comité Estatal de Planeación Para el Desarrollo de Oaxaca. Available from http// www.coplade.oaxaca.gob.mx/?. [Last accessed on 2019 on Jan 06].

Crespo, G. M. R., E. D. R. González, M. R. Rodríguez, L. A. Rendón, S. J. I. Del Real Laborde and M. J. P. Torres. 2013. Evaluación de la composta de bagazo de agave como componente de sustratos para producir plántulas de agave azul tequilero. Rev. Mex. Cienc. Agríc. 4 1161-1173.

DOF. 2002. Norma Oficial Mexicana NOM-021-RECNAT-2000. Que Establece las Especificaciones de Fertilidad, Salinidad y Clasificación de Suelos. Estudios, Muestreo y Análisis, Diario Oficial de la Federación. Available from http//www.diariooficial. gob.mx/nota_detalle.php?codigo=717582\&fecha=31/12/2002. [Last accessed on 2018 Feb 08].

Eguiarte, L. E., E. Aguirre-Planter, X. Aguirre, R. Colín, A. González, M. Rocha, E. Scheinvar, L. Trejo and V. Souza. 2013. From isozymes to genomics Populations genetics and conservation of Agave in México. Bot. Rev. 79 483-506.

Enríquez-del Valle, J. R. 2008. La Propagación y Crecimiento de Agaves. Fundación Produce Oaxaca A.C. Instituto Tecnológico del Valle de Oaxaca, Oaxaca, p. 46.

Enríquez-del Valle, J. R., A. S. Estrada, G. Rodríguez-Ortiz, V. A. Velasco-Velasco and G.V. Campos-Ángeles. 2013. Sustrato y dosis de fertirriego en la aclimatización de vitroplantas de Agave americana var. oaxacencis. Rev. Facult. 45 341-348.

Enríquez-del Valle, J. R., V. A. Velasco-Velasco, G.V. CamposÁngeles, E. Hernández-Gallardo and M. N. Rodríguez-Mendoza. 2009. Agave angustifolia plants grown with different fertigation doses and organic substrates. Acta Hortic. 843 441-348.

Espinosa, H. P., L. P. López, V. C. Arredondo and J. R. Enríquez-del Valle. 2005. Cuáles son los métodos para obtener la planta de maguey que necesitamos para establecer nuestra plantación en campo. In M. del Magueyero (Ed.), Comisión de trabajo para el desarrollo responsable de la industria del maguey y del mezcal en Oaxaca. Consejo Oaxaqueño del Maguey y del Mezcal, Oaxaca, p. 3-42.

Faiss, M., J. Zalubilová, M. Strnad and T. Schmülling. 1997. Conditional transgenic expression of the ipt gene indicates a function for cytokinins paracrine signaling in whole tobacco plants. Plant J. 12 401-415.

García-Herrera, E. J., S. J. Méndez-Gallegos and D. TalaveraMagaña. 2010. El género agave spp. en México Principales usos de importancia socioeconómica y agroecológica. VIII Simposium-taller nacional y 1er internacional "producción y aprovechamiento de nopal". Rev. Salud Pública Nutr. Ed. Especial. 5 109-129.

García-Mendoza, A. J. 2007. Los agaves de México. Ciencias. 87 14-23.

García-Moya, E., A. Romero-Manzanares and P. S. Nobel. 2011. Highlights for agave productivity. Glob. Change Biol. Bioenergy. 3 1-14.

George, E. F., M. A. Hall and G. Klerk. 2008. Plant Propagation by Tissue Culture An Plant Growth Regulators I. $3^{\text {rd }}$ ed. Springer, Netherlands, pp. 175-204.

Infante, D., G. González, L. Peraza-Echeverría and M. Keb-Llanes. 2003. Asexual genetic variability in Agave forcroydes. Plant Sci. 164 223-230.

Lara-Ávila, J. P. and A. G. Alpuche-Solís. 2016. Análisis de la biodiversidad genética de agaves mezcaleros del centro de México. Rev. Fitotec. Mex. 39 323-330.

López, C. Y. J., G. A. G. Martinez and A. G. Caballero. 2016. Diagnostico de la Situacion Agave-mezcal y Opciones de Desarrollo Local en Comunidades Indígenas de Oaxaca. In AMECIDER ITM. $21^{\text {st }}$ Encuentro Nacional Sobre el Desarrollo Regional, Yucatán, p. 19.

Martínez, S. T., C. T. Méndez, L. V. V. Echegaray and L. A. Martínez. 2013. Diagnóstico Sistema Producto Maguey Mezcal. Universidad Autónoma, Chapingo, p45-49.

Martínez-Aguilar, J. F. and A. Peña-Álvarez. 2009. Characterization of five typical agave plants used to produce mezcal through their simple lipid composition analysis by gas chomatography. J. Agric. Food Chem. 57 1933-1939.

Martínez-Ramírez, S., A. Trinidad-Santos, G. Bautista-Sánchez and E. C. Pedro-Santos. 2013. Crecimiento de plántulas de dos especies de mezcal en función del tipo de suelo y nivel de fertilización. Rev. Fitotec. Mex. 23 387-393.

Miguel, L. M. E., J. R. Enríquez-del Valle, V. A. Velasco-Velasco, A. Y. Villegas and J. C. Carrillo-Rodríguez. 2014. Concentración de benciladenina, tipo y dosis de carbohidratos en el medio de cultivo para la proliferación de brotes de Agave americana. Rev. Facult. 46 97-107.

Mok, M. C., R. C. Martin and S. W. D. Mok. 1999. Cytokinins Biosynthesis, metabolism and perception. In Vitro Cell. Dev. Biol. Plant. 36 102-107.

Nobel, P. S. 1977. Water relations of flowering of Agave deserti. Bot. Gazette. 138 1-6.

Nobel, P. S. 1989. A nutrient index quantifying productivity of agaves and cacti. J. Appl. Ecol. 26 635-645.

Nolasco, C. H. 2015. Informe de la producción 2014. Consejo Regulador del Mezcal Denominación de Origen. Consejo Regulador del Mezcal, Oaxaca, México, p. 74.

Palma, F., P. Pérez and V. Meza. 2016. Diagnóstico de la Cadena de Valor Mezcal en Las Regiones de Oaxaca. Available from http// www.coplade.oaxaca.gob.mx/wp-content/uploads/2017/04/ Perfiles/AnexosPerfiles/6.\%20CV\%20MEZCAL.pdf. [Last 
accessed on 2018 Mar 15].

Ríos-Ramírez, S. C., J. R. Enríquez-del Valle, G. Rodríguez-Ortiz, and J. Ruiz-Luna. 2017. Benzylaminopurine and indol-3-acetic acid concentrations in in vitro proliferation of Agave angustifolia adventitious shoots. Cien. Investig. Agric. 44 285-294.

SAS Institute. 2004. SAS 9.0 SQL Proceduce User`s Guide. Cary, NC, USA.

Silva-Montellano, A. and L. E. Eguiarte. 2003. Geographic patterns in the reproductive ecology of Agave lechuguilla (Agavaceae) in the Chihiahuan desert. I. Floral characteristics, visitors, and fecundity. Am. J. Bot. 90 377-387.

Steiner, A. A. 1984. The Universal Nutrient Solution. International Society for Soilless Culture. $6^{\text {th }}$ International Congress on Soilless Culture, Luteren, Wageningen, pp. 633-650.

Szarek, S. R. and G. E. Holmesley. 1996. Physiological activity in persistent bulbils of Agave vilmoriniana (Agavaceae). Am. J. Bot. 83 903-909.

Trame, A. M., A. J. Coddington and K. N. Paige. 1995. Field and genetic studies testing optimal outcrossing in Agave schottii, a long-lived clonal plant. Oecologia. 104 93-100.

Trejo-Téllez, L. I., N. I. Torres-Flores, O. Tejeda-Sartorius, B. I. Trejo-Téllez, M. Ramírez-Martínez and F. C. GómezMerino. 2013. Nitrógeno y potasio en la acumulación de biomasa en dos especies de alcatraz. Rev. Mex. Cienc. Agríc. 5 1063-1068.

Verduzco-Martínez, J., C. Pedro-Rojas and R. Mercado-Hernández. 2009. Caracterización e identificación taxonómica del maguey. Rev. Salud Públic. Nutr. 2 75-76.

Zizumbo-Villareal, D., O. Vargas-Ponce, J. J. Rosales-Adame and P. Colunga-GarcíaMarin. 2013. Sustainability of the traditional management of Agave genetic resources in the elaboration of mescal and tequila spirits in Western Mexico. Genet. Resour. Crop Evol. 60 33-47.

Zúñiga-Estrada, L., E. R. Rosales, M. J. Yáñez-Morales and C. Jacques-Hernández. 2018. Características de una planta MAC, Agave tequilana desarrollada con fertigación en Tamaulipas, México. Rev. Mex. Cienc. Agríc. 9 553-564. 